\title{
Modelling the clumping-induced polarimetric variability of hot star winds
}

\author{
B. Davies ${ }^{1,2}$, J. S. Vink ${ }^{3,4,5}$, and R. D. Oudmaijer ${ }^{2}$ \\ 1 Center for Imaging Science, Rochester Institute of Technology, 84 Lomb Memorial Drive, Rochester, NY 14623, USA \\ e-mail: davies@cis.rit.edu \\ 2 School of Physics \& Astronomy, University of Leeds, Woodhouse Lane, Leeds LS2 9JT, UK \\ 3 Imperial College, Blackett Laboratories, Prince Consort Road, London SW7 2BZ, UK \\ 4 Lennard-Jones Laboratories, Keele University, Staffordshire ST5 5BG, UK \\ 5 Armagh Observatory, College Hill, Armagh BT61 9DG, NI, UK
}

Received 30 January 2007 / Accepted 12 April 2007

\begin{abstract}
Context. Clumping in the winds of massive stars may significantly reduce empirical mass-loss rates, and which in turn may have a large impact on our understanding of massive star evolution.

Aims. Here, we investigate wind-clumping through the linear polarization induced by light scattering off the clumps.

Methods. Through the use of an analytic wind clumping model, we predict the time evolution of the linear polarimetry over a large parameter space. We concentrate on the Luminous Blue Variables, which display the greatest amount of polarimetric variability and for which we recently conducted a spectropolarimetric survey.

Results. Our model results indicate that the observed level of polarimetric variability can be reproduced for two regimes of parameter space: one of a small number of massive, optically-thick clumps; and one of a very large number of low-mass clumps.

Conclusions. Although a systematic time-resolved monitoring campaign is required to distinguish between the two scenarios, we currently favour the latter, given the short timescale of the observed polarization variability. As the polarization is predicted to scale linearly with mass-loss rate, we anticipate that all hot stars with very large mass-loss rates should display polarimetric variability. This is consistent with recent findings that intrinsic polarization is more common in stars with strong $\mathrm{H} \alpha$ emission.
\end{abstract}

Key words. stars: mass-loss - stars: winds, outflows - polarization - techniques: polarimetric - stars: early-type - stars: supergiants

\section{Introduction}

Many different species of massive star display intrinsic linear polarization - from main-sequence O stars (Lupie \& Nordsieck 1987, LN87 hereafter), to post main-sequence transitional objects such as OB supergiants (Hayes 1984a; LN87), Red Supergiants (Hayes 1984b), Luminous Blue Variables (LBVs, Davies et al. 2005), B[e] supergiants (Melgarejo et al. 2001) and Wolf-Rayet stars (WRs, St.-Louis et al. 1987). This intrinsic polarization results from the scattering of starlight by aspherical structure in the stellar wind. In the case of the B[e]SGs, this inhomogeneity is thought to be in the form of a circumstellar disk, although the data for this is limited (e.g. Zickgraf \& SchulteLadbeck 1989; Schulte-Ladbeck et al. 1993). The intrinsic polarization of the LBV AG Carina was also thought to result from a global axi-symmetric wind structure (Schulte-Ladbeck et al. 1994). However, multi-epoch observations have shown that the polarization angle of AG Car is stochastically variable (Davies et al. 2005). The polarization of P Cygni was found to be variable on timescales of $\lesssim$ weeks (Nordsieck et al. 2001), which is similar to the short timescale variability observed in WRs (Robert et al. 1989). This behaviour is commonly explained as being due to wind-clumping (e.g. LN87).

Not just a quirk of massive stars, wind-clumping could have serious implications for our understanding of stellar evolution. Mass-loss rates have traditionally been determined by fitting recombination lines with models that assume a smooth outflow (for LBVs see e.g. Stahl et al. 2001; Machado et al. 2002). As recombination is a two-body interaction process it depends on $\rho^{2}$, so inhomogeneity will result in an over-estimation of the density (as $\left\langle\rho^{2}\right\rangle /\langle\rho\rangle^{2}$ ), and hence an over-estimation of the massloss rate.

Recently, there has been much discussion about clumping in the winds of $\mathrm{O}$ stars. The value of the clumping factor $f_{\mathrm{cl}}=\left\langle\rho^{2}\right\rangle /\langle\rho\rangle^{2}$ is as yet an open issue. On the one hand, $\mathrm{H} \alpha$ analyses by Repolust et al. (2004) and Mokiem et al. (2007) find that a modest amount of wind clumping, with a clumping factor up to $f_{\mathrm{cl}} \simeq 5$ is required to match the smooth line-driven wind models of Vink et al. (2000). But on the other hand, X-ray (e.g. Oskinova et al. 2006) and quantitative UV studies (e.g. Bouret et al. 2005; Fullerton et al. 2006) call for $f_{\mathrm{cl}}$ values much larger than 10. If confirmed, this could significantly affect our understanding of massive star evolution.

In addition to spectral diagnostic techniques, linear polarimetry studies have proven to be a powerful tool to probe the geometry of the wind structure. For instance, the results of a spectropolarimetric survey of LBVs by Davies et al. (2005) call for an onset of wind clumping in close proximity to the stellar photosphere, as to be able to reproduce the observed levels of polarization. LBVs may be an ideal testbed to study hot star wind clumping as the strength of the intrinsic polarization is much larger than that observed in O and WR stars. Systematic monitoring campaigns have shown that WRs display polarization around the $0.1 \%$ level, with variability on very short timescales of $<1$ day. Additionally, the variability was found to have a greater amplitude for those stars with slower terminal 
wind speeds $\left(v_{\infty}=1200 \rightarrow 3600 \mathrm{~km} \mathrm{~s}^{-1}, \Delta P \sim 0.1 \rightarrow 0.02 \%\right.$; Robert et al. 1989). LBVs have wind speeds around a factor of 10 slower and show intrinsic polarization of $\gtrsim 0.5 \%$. Although there have yet to be any systematic polarimetric monitoring campaigns of LBVs, the data of Hayes (1985) appeared to indicate variability on timescales comparable to the wind flow-time $\left(t_{\mathrm{fl}} \equiv R_{\star} / v_{\infty}\right)$, which for LBVs is on the order of $\sim$ days. This is consistent with the polarization arising within a few stellar radii of the central star (cf. Cassinelli et al. 1987).

Theoretical studies of clumping-induced polarization are few, and tend to be "first-step" investigations rather than comprehensive studies. Rodrigues \& Magalhães (2000) and Code \& Whitney (1995) presented Monte-Carlo studies of the polarization produced by a single clump. Harries (2000) studied the time-variability of a clumpy wind, again with a Monte-Carlo code, but did not investigate the effect of changing stellar/wind properties, nor the polarimetric variability on longer timescales. Both of these studies concluded that the clumps must be optically thin, as increasing the clump optical depth begins to have a conspicuous effect on time-resolved observations of spectral line-profiles. Li et al. (2000) used an analytical model to investigate the polarimetric variability of WRs, but again did not study the effect of different stellar parameters. Richardson et al. (1996) studied specifically the effect of a clumpy wind on the ratio of photometric to polarimetric variability in the case of WRs. Brown et al. (2000) investigated the "redistribution" of a smooth outflow into clumps, simulating the pile-up of material caused by e.g. shocks in the wind. They concluded that small-scale redistribution close to the star could not produce significant levels of polarization. This implies that clumps producing shorttimescale polarimetric variability, which are necessarily close to the star, must be localized over-densities of ejecta, as opposed to the result of a smooth outflow being "snow-ploughed" by a shock-front.

In this paper we use an analytic model to build on these previous studies and fully investigate the polarimetric variability produced by a clumpy wind across a range of stellar parameters typical of massive evolved stars. We place particular emphasis on LBVs - the group of stars which display the strongest polarimetric variability. A full description of the model is presented in Sect. 2, along with a few basic applications to geometries explored in other work to serve as validation. The relevant input parameters are discussed in Sect. 3, leading to the model results and discussion in Sects. 4 and 5.

\section{Description of the model}

In this analytic study we follow the approach of, amongst others, Brown \& McLean (1977); Brown et al. (2000); Li et al. (2000). The strategy for the construction of the model is as follows: the polarization due to a single clump is determined as a function of distance from the illuminating star. Through the velocity-law of the wind, an expression for the polarization as a function of time is found. Multiple clumps are added, and the total polarization found from the vector sum of the polarization of each individual clump. The clumps are then given a typical ejection timescale, and the polarization as a function of time is calculated as new clumps are ejected and move through the wind. After the simulation reaches a steady-state, the polarization is monitored for a further period of time to determine the typical polarization level and its variability.

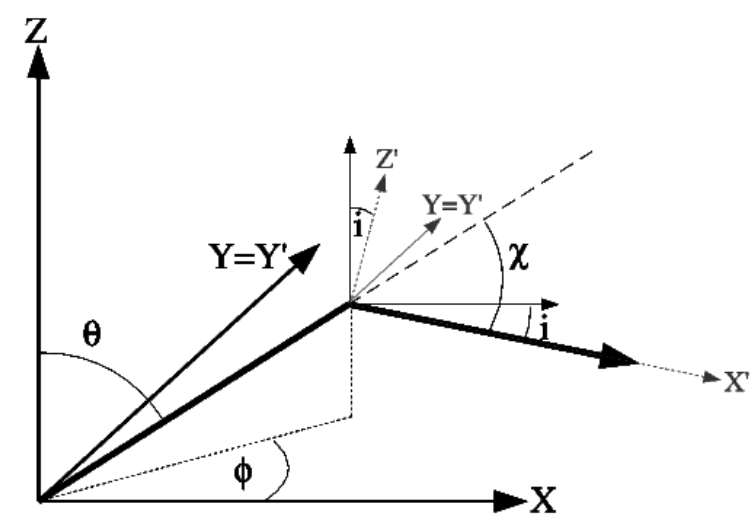

Fig. 1. The coordinate system used in this model, as defined in Brown $\&$ McLean (1977). A beam with direction $(\theta, \phi)$ scatters through an angle $\chi$ towards the observer. The observer's reference frame is rotated through an angle $i$ with respect to the star's reference frame.

\subsection{Model geometry}

The coordinate system used is that defined in Brown \& McLean (1977), and is illustrated in Fig. 1. The angle of the plane of scattering to the observer $\chi$ can be described in terms of the polar $(\theta)$ and azimuthal $(\phi)$ angles of the star's reference frame, and the inclination of this frame to the plane of the sky $i$ :

$\cos \chi=-\cos \theta \cos i+\sin \theta \sin i \cos \phi$.

The position angle (PA) of the polarization, $\Theta$, is described by,

$\sin \Theta=\frac{\cos \theta \sin i+\sin \theta \cos i \cos \phi}{\left\{(\cos \theta \sin i+\sin \theta \cos i \cos \phi)^{2}+\sin ^{2} \theta \sin ^{2} \phi\right\}^{0.5}}$.

\subsection{Polarization of axi-symmetric material}

From Brown et al. (2000), which uses the expressions of Brown \& McLean (1977) and Cassinelli et al. (1987), the polarization produced by electron scattering material in a stellar wind as a function of distance to the star $r$, polar angle $\theta$, and azimuthal angle $\phi$, is described by,

$$
\begin{array}{r}
P(\mu, r)=\frac{3}{16} \sigma_{\mathrm{T}} \sin ^{2} \chi \int_{\mu_{1}}^{\mu_{2}}\left(1-3 \mu^{2}\right) \mathrm{d} \mu \\
\times \int_{r_{1}}^{r_{2}} n_{\mathrm{e}}(r) \sqrt{1-\left(\frac{R_{\star}}{r}\right)^{2}} \mathrm{~d} r
\end{array}
$$

where $\sigma_{\mathrm{T}}$ is the Thompson scattering cross-section, $\chi$ is the angle between the plane of scattering and the observer, $\mu=\cos \theta$, $R_{\star}$ is the radius of the star, and $n_{\mathrm{e}}$ is the electron number density which in this case is a function of $r$ only. The square-root factor within the $r$ integral is known as the finite-star correction-factor (Cassinelli et al. 1987), and accounts for the finite stellar "disk" seen by the scattering material. This formalism can be used to determine the polarization produced by different scattering geometries, and an illustration of this can be seen in Fig. 2.

\subsection{Clumps}

The amount of polarization a clump produces is determined by how much light is intercepted, as well as the clump's size and morphology. Arbitrary changes in clump morphology can produce minor changes in $P$ if the clump is close to the star, due to 


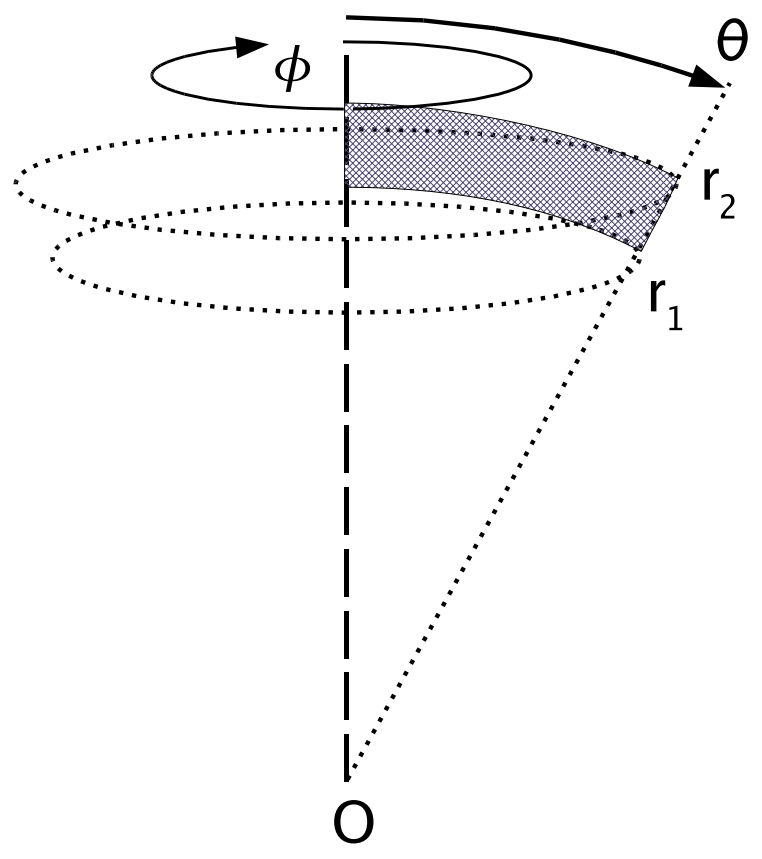

Fig. 2. The geometry assumed in Eq. (3). The electron density is assumed to be symmetric about the central axis (i.e. over all $\phi$ ), such that by specifying the limits $r_{1}$ to $r_{2}$ and $\theta_{1}$ to $\theta_{2}$ we are integrating over the volume swept out by the shaded area, in this case a "shell".

the effect of the finite size of the illuminating star. This effect is small, however, and it is a reasonable approximation to say that, for a constant column-density, halving a clump's size (and hence doubling its density) produces no difference in polarization (see also Brown et al. 2000). For this reason we concentrate on the simplest morphology for the clumps, that of constant angular size and thickness. In a radiatively driven wind this scenario is plausible, as each particle in the wind is driven radially outwards, and any inhomogeneity will grow in size with the square of the distance.

\subsubsection{Polarization of a single "shell" clump}

The geometry of Fig. 2 and Eq. (3) are used as a starting point. The angular size remains constant with distance from the star, so $\mu$ can be integrated from $\mu_{1}=0$ to $\mu_{2}=1-\Delta \Omega / 2 \pi$, where $\Delta \Omega$ is the angular size of the clump. The electron number density is parameterized in order to conserve mass for a clump with constant angular size, thickness and number of electrons, i.e.

$n(r)=n_{0}\left(\frac{R_{\star}}{r}\right)^{2}$

where $n_{0}$ in the initial number density of the clump at $r=R_{\star}$.

Substituting Eq. (3) into Eq. (3) and setting $x=r / R_{\star}$, the polarization of a single clump at a distance $x^{\prime}$ from a star is,

$$
\begin{aligned}
P= & \frac{3}{16} \sigma_{\mathrm{T}} n_{0} R_{\star} \sin ^{2} \chi\left(\mu_{2}-\mu_{2}^{3}\right) \\
& \times \int_{x^{\prime}}^{x^{\prime}+\Delta r} \frac{1}{x^{2}} \sqrt{1-\frac{1}{x^{2}}} \mathrm{~d} x
\end{aligned}
$$

where $\Delta r$ is the dimensionless thickness of the shell.

As this is an analytical study, multiple scattering is not taken into account in this equation. When multiple scattering effects start to become relevant, i.e. when $\tau_{\mathrm{e}}>1$, the results from Eq. (4) would become unphysical. To assess the importance of multiple scattering, we need to monitor the optical depth, and we re-write Eq. (4) in terms of the initial optical depth per clump $\tau_{0}$, i.e. when $r=R_{\star}$ :

$$
\begin{aligned}
\tau_{0} & =\frac{3}{16} \sigma_{\mathrm{T}} \int_{r_{1}}^{r_{2}} n(r) \mathrm{d} r \\
& =\frac{3}{16} \sigma_{\mathrm{T}} n_{0} R_{\star} K
\end{aligned}
$$

where,

$$
\begin{aligned}
K & =\int_{1}^{1+\Delta r} \frac{1}{x^{2}} \mathrm{~d} x \\
& =1-\frac{1}{1+\Delta r} \\
& \simeq \Delta r(\text { if } \Delta r \ll 1) .
\end{aligned}
$$

In order to relate the initial optical depth to other observables, the initial optical depth per clump can be expressed in terms of the stellar parameters. The mass-loss rate and clump ejection rate, $\dot{N}$, are related via,

$\dot{M}=\dot{N} N_{\mathrm{e}} \mu_{\mathrm{e}} m_{\mathrm{H}}$

where $N_{\mathrm{e}}$ is the number of electrons in each clump, $\mu_{\mathrm{e}}$ is the mean mass-per-particle and $m_{\mathrm{H}}$ is the mass of a proton. To parameterize the ejection rate in the general case, the formalism of $\mathrm{Li}$ et al. (2000) is adopted by defining the ejection rate per wind flow-time $\mathcal{N}$, where $t_{\mathrm{fl}} \equiv R_{\star} / v_{\infty}$ :

$\mathcal{N}=\dot{N} t_{\mathrm{fl}}=\dot{N} R_{\star} / v_{\infty}$

Therefore, the number of electrons in each clump:

$N_{\mathrm{e}}=\frac{\dot{M} R_{\star}}{\mu_{\mathrm{e}} m_{\mathrm{H}} \mathcal{N} v_{\infty}}$

The initial volume per clump is,

$V_{\mathrm{cl}}=\Delta \Omega R_{\star}^{3} \Delta r$

where $\Delta \Omega$ is the solid angle $2 \pi\left(1-\mu_{2}\right)$, and $\Delta r$ is the clump thickness in units of $R_{\star}$ and is assumed to be $\ll 1$. The electron density per clump is then,

$n_{0}=\frac{N_{\mathrm{e}}}{V_{\mathrm{cl}}}=\frac{\dot{M}}{\mu_{\mathrm{e}} m_{\mathrm{H}} \mathcal{N} v_{\infty} \Delta \Omega R_{\star}^{2} \Delta r}$.

Equation (4) can now be re-written as,

$P=\frac{\tau_{0}}{\Delta r} \sin ^{2} \chi\left(\mu_{2}-\mu_{2}^{3}\right) \int_{x^{\prime}}^{x^{\prime}+\Delta r} \frac{1}{x^{2}} \sqrt{1-\frac{1}{x^{2}}} \mathrm{~d} x$

where,

$\frac{\tau_{0}}{\Delta r}=\frac{3}{16} \sigma_{\mathrm{T}} \frac{\dot{M}}{\mu_{\mathrm{e}} m_{\mathrm{H}} \mathcal{N} v_{\infty} \Delta \Omega R_{\star} \Delta r}$.

The results of the polarization of a single clump as a function of distance are shown for different optical depth values $\tau$ in Fig. 3. 


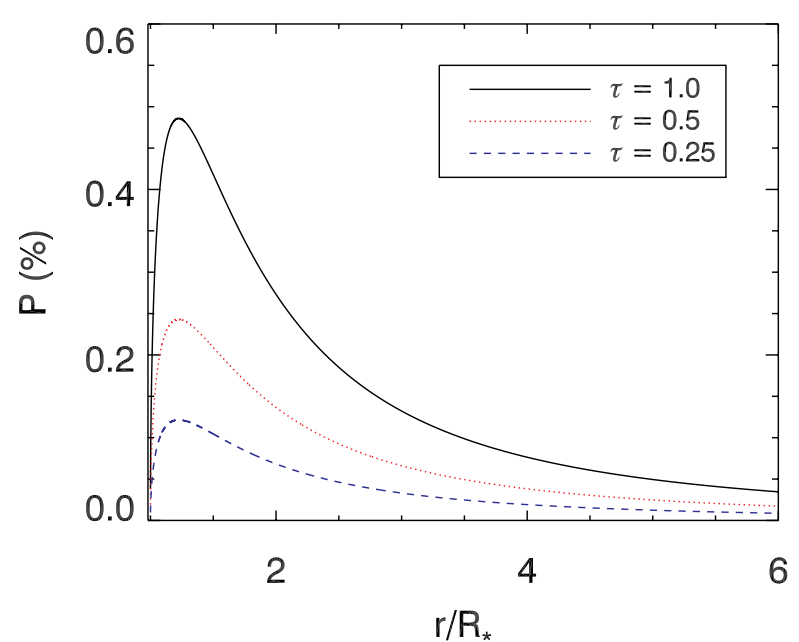

Fig. 3. The polarization as a function of distance for a single clump for three values of $\tau$. The clump size is $\Delta \Omega=0.04, \Delta r=0.01$; and $\sin ^{2} \chi=1$. The polarization falls-off as roughly $1 / r$, with the attenuation at small $r$ due to the finite size of the illuminating star. It can be seen that at distances of $r / R_{\star} \sim 5$ the polarization has already decreased from the maximum value by a factor of $\sim 10$.

\subsubsection{Polarization of a single clump as a function of time}

Having determined the polarization as a function of distance from the central star, we calculate the time behaviour of the polarization. The velocity of the wind as a function of distance from the star is described by the so-called "beta-law",

$v(r)=v_{\infty}\left(1-\frac{b R_{\star}}{r}\right)^{\beta}$

where $v_{\infty}$ is the terminal velocity of the wind, $b$ is a constant which dictates the initial velocity of the wind $v_{0}$ (i.e. at $r=R_{\star}$ ), and $\beta$ is a constant which determines the wind's acceleration. Hot supergiants are typically thought to have $1<\beta<2$ (see e.g. Herrero et al. 2002), so for ease of calculation a value of $\beta=1$ is used throughout most of this study. By rearranging Eq. (10), setting $\beta=1$, and substituting $x=r / R_{\star}$, we find an expression for the time since ejection $t$ as a function of distance $x$ and the velocity-law parameters $v_{\infty}, \beta$, and $b$,

$t=\int \frac{\mathrm{d} r}{v(r)}=\frac{R_{\star}}{v_{\infty}}[x+b \ln (x-b)+c]$

where $c$ is a constant of integration, which is determined by forcing $x=1$ at $t=0$. The polarization as a function of time can now be calculated by solving Eqs. (4) and (11) parametrically. The initial velocity $v_{0}$ is set to the isothermal sound speed at the temperatures considered. Altering $v_{0}$ by a factor of two was found to have little difference on the results of a given simulation, so for simplicity the value $v_{0}=10 \mathrm{~km} \mathrm{~s}^{-1}$ is used throughout this study.

The effect of increasing $\beta$ is shown in Fig. 4. The plot shows the polarization as a function of time for three values of $\beta$, and two separate ratios of $v_{0} / v_{\infty}$. As increasing $\beta$ increases the time the clump spends in the inner regions of the wind (the "dwell time"), the polarization remains high for longer. The effect is more pronounced when $v_{0} / v_{\infty}$ is higher.

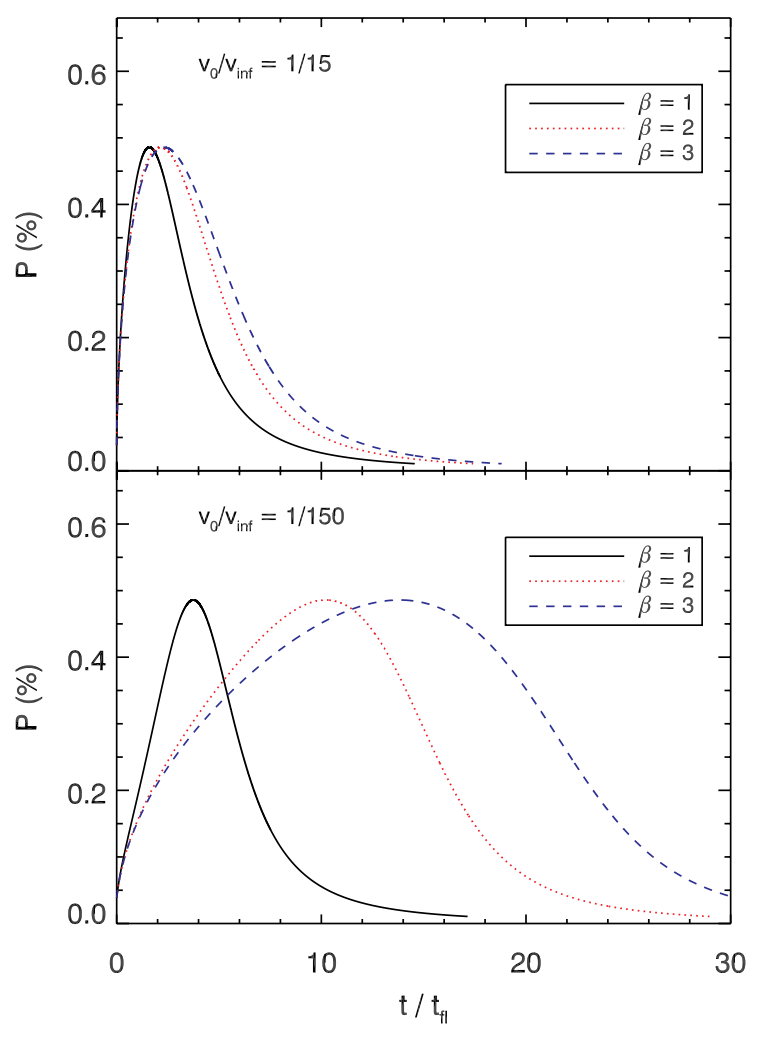

Fig. 4. The polarization as a function of time (in units of wind flowtime) for three different values of $\beta$. Top: for slow terminal wind speed; Bottom: fast terminal wind speed. Same clump parameters are used as for Fig. 3.

\subsubsection{Polarization of multiple clumps}

Clumps are ejected radially from a random location on the stellar surface, with $0<\phi_{j}<2 \pi$ and $-1<\cos \theta_{j}<1$ for the $j$ th clump. For a given inclination angle, the polarization and its position angle due to a single clump as a function of time are calculated through Eqs. (1), (2), (9a), and (11). Clumps are ejected over a typical timescale $T_{\text {ej }}$, such that $T_{\text {ej }}=R_{\star} / \mathcal{N} v_{\infty}$. The effect of randomizing $T_{\mathrm{ej}}$ was experimented with, such that $T_{\mathrm{j}}=T_{\mathrm{ej}} \pm$ $\sigma_{\mathrm{ej}}$. This was found to have no effect on the results, and was computationally more expensive as it required finer grids to be set up. Consequently, a uniform $T_{\mathrm{ej}}$ was used throughout each simulation.

To calculate the total polarization due to all clumps at any one time, the polarization due to each clump must be converted into the linear Stokes parameters,

$Q=P \cos 2 \Theta$

$U=P \sin 2 \Theta$

where $\Theta$ is perpendicular to the plane of scattering. The total polarization at any one time is then determined from the sum of the Stokes vectors of each of the $N$ clumps in the wind,

$P_{\text {tot }}^{2}=\left(\sum_{j=1}^{N} Q_{j}\right)^{2}+\left(\sum_{j=1}^{N} U_{j}\right)^{2}$
$\Theta_{\mathrm{tot}}=\frac{1}{2} \tan ^{-1}\left(\sum_{j=1}^{N} U_{j} / \sum_{j=1}^{N} Q_{j}\right)$. 
In order to determine the typical polarization of a model run, the simulation is run until it reaches an equilibrium, and the first clumps to be ejected no longer contribute significantly to the overall polarization. This occurs when the first clumps reach $r / R_{\star} \gtrsim 6$ (see Fig. 3). The polarization is then still monitored for a further 30 flow-times, and the time-averaged polarization $\langle P\rangle$ over this period, together with the rms variability $\Delta P$, are recorded. As the simulation is stochastic by design, it can sometimes throw up atypical results. To compensate for this, the process is repeated several times for each set of parameters, and the mean is taken. The experimental uncertainties in $\langle P\rangle$ and $\Delta P$ were taken to be $\sigma / \sqrt{n}$, where $\sigma$ is the rms scatter of the results of $n$ simulations.

\subsubsection{High ejection-rates}

The goal of this work is to reproduce the polarimetric variability of hot stars - both on long timescales, as found by the repeated measurements in the literature; and short-timescales, as found by e.g. Hayes (1984a) and St.-Louis et al. (1987). At low ejection rates, individual ejection events may be resolvable by nightly monitoring, and the model would have to be dynamic to simulate this, i.e. follow the polarimetric behaviour in real-time as clumps move through the wind, whilst new clumps are ejected.

However, at high ejection rates the distribution of clumps close to the photosphere will look completely different from night-to-night, and so will the polarization. In this situation, there is nothing to be gained from computationally-expensive dynamical modelling as ejection events are unresolvable. In the high- $\mathcal{N}$ regime, calculations are therefore performed using a static model. Here, as the polarization at each observation is unrelated to the last, a series of wind "snap-shots" are generated, which represent a sample of independent observations. Clump distances are randomly-generated according to the ejection rate and velocity law, and the total polarization is calculated. This is repeated $10^{3}$ times, and $\bar{P}$ and $\Delta P$ calculated.

Whilst being unable to predict the time-evolution of individual clumps, this static method has the advantage of being able to simulate a much broader range of $\mathcal{N}$ values - at drastically reduced computation times.

\subsection{Optically-thick clumps}

Equation (3) shows that, in this study, the amount of polarization produced by a clump is proportional to the clump's optical depth, as denser clumps scatter more light.

However, this will only hold as long as the clump is optically thin. When the optical depth becomes larger than $\sim 1$, multiple scattering becomes important. In this regime, the relation in Eq. (3) breaks down as a photon which is scattered twice cannot produce twice as much polarization.

To investigate the effect of optically-thick clumps, a firstorder approximation is made from the Monte-Carlo work of Rodrigues \& Magalhães (2000). They studied the polarization produced by starlight scattering off clumps with optical depths as high as $\tau_{\mathrm{e}}=10$. Their results show that, in the low- $\tau_{\mathrm{e}}$ regime, the polarization rises almost linearly with optical depth, until $\tau$ reaches $\sim 1$. At high- $\tau_{\mathrm{e}}$ the polarization flattens off. This can be understood in terms of multiply-scattered photons "forgetting" where they came from, with only the singly-scattered photons carrying the scattering geometry information. This explains the slight fall-off of $P$ with high $\tau$, as less and less of the observed photons are singly-scattered.
We approximate the results of Rodrigues \& Magalhães (2000) such that polarization is linear with optical depth up to $\tau=1$, and then constant for higher $\tau$. In this situation, as an optically-thick clump moves away from the star its density decreases as $1 / r^{2}$, but the polarization remains constant while the optical depth is larger than 1 . When the clump reaches a distance such that $\tau=1$, its polarization decreases rapidly in the same manner as an optically-thin clump.

In making this approximation, we note that both Code \& Whitney (1995) and Rodrigues \& Magalhães (2000) considered the case of spherical clumps, while in this work we consider only flattened, "shell" clumps. Although we argue in Sect. 2.3 that clump shape is a redundant parameter in the optically-thin limit, the relative invariance of clump morphology to polarization may not hold for optically-thick clumps. For example, in the case of a spherical clump, the limb will always be optically-thin to some extent, whereas the optical depth of a "shell" clump will always be the same along all radial paths from the star. This problem bears some similarity to the challenges faced when modelling clouds in planetary atmospheres (van de Hulst 1980). The impact and limitations of our approximation in the context of the results will be discussed further in Sect. 4.4.

\section{Model parameters}

For a given mass-loss rate and ejection timescale, the key input parameters are: the terminal wind velocity, the clump size and optical depth, the stellar radius, and the ejection timescale. Each of these parameters are discussed below.

\subsection{Clump size}

The size of each clump is determined by its angular size $\Delta \Omega$ and its thickness $\Delta r$. Provided $\Delta r$ is small, it has no effect on the polarization per clump as the optical depth remains the same. Varying $\Delta \Omega$ has a small effect, as for large $\Delta \Omega$ the polarization at the edge of a shell slightly cancels that of the opposite side. This can be seen in the simulations (Sect. 4.2.2).

\subsection{Terminal wind velocity $v_{\infty}$, and stellar radius $R_{\star}$}

The polarization produced by a single clump falls off quickly with distance to the star (see Fig. 3). The amount of time that each clump will spend close to the star (the "dwell time") is therefore a major factor in the overall level of polarization. The dwell time is affected not only by speed of the outflow, but also the size of the star. This is therefore reflected in the star's wind flow-time $t_{\mathrm{fl}} \equiv R_{\star} / v_{\infty}$.

The terminal velocity is typically a few times the stellar escape velocity, which in turn depends on the effective gravity of the star:

$g_{\mathrm{eff}}=g_{\mathrm{N}}-g_{\mathrm{rad}}$

where $g_{\mathrm{N}}$ is the Newtonian gravity $G M / R_{\star}^{2}$ and $g_{\text {rad }}$ is the outward radiative acceleration due to the continuum opacity of free electrons in the ionised stellar atmosphere,

$g_{\mathrm{rad}}=\frac{\kappa_{\mathrm{e}} L_{\star}}{4 \pi R_{\star}^{2} c}$ 
The effective mass is then defined as,

$M_{\text {eff }}=\left(1-\Gamma_{\mathrm{e}}\right) M_{\star}$

where $\Gamma_{\mathrm{e}}=g_{\mathrm{rad}} / g_{\mathrm{N}}$. The terminal wind velocity is now,

$$
\begin{aligned}
v_{\infty} & =Q_{\mathrm{BSJ}} v_{\mathrm{esc}} \\
& =Q_{\mathrm{BSJ}} \sqrt{\frac{2 G M_{\mathrm{eff}}}{R_{\star}}}
\end{aligned}
$$

where $Q_{\mathrm{BSJ}}$ is the "bi-stability jump" ratio which is predicted to depend on the star's effective temperature (Vink et al. 1999). According to empirical studies (Lamers et al. 1995; Kudritzki \& Puls 2000), $Q_{\text {BSJ }}$ takes two distinct values,

$$
\begin{aligned}
& Q_{\mathrm{BSJ}} \simeq 1.3 \quad\left(T_{\text {eff }} \lesssim 21000 \mathrm{~K}\right) \\
& Q_{\mathrm{BSJ}} \simeq 2.5\left(T_{\mathrm{eff}} \gtrsim 21000 \mathrm{~K}\right) .
\end{aligned}
$$

As the stellar radius and effective temperature are related via,

$L_{\star}=4 \pi R_{\star}^{2} \sigma_{\mathrm{B}} T_{\mathrm{eff}}^{4}$

the terminal wind velocity can be found from the star's mass, luminosity and temperature. In the case of LBVs, this allows us to predict how the terminal wind-speed varies over the S Doradus phases which are thought to occur at constant bolometric luminosity.

We note that a recent study of blue supergiants over the bistability regime indicates that $Q_{\mathrm{BSJ}}$ may not be a step-function, but instead it may decrease smoothly with decreasing temperature (Crowther et al. 2006). The binning of the data by effective temperature by Lamers et al. may give the impression that $Q_{\mathrm{BSJ}}$ behaves like a step function. One of the main reasons $Q_{\mathrm{BSJ}}$ may not be a step function is that one is comparing objects with very different stellar parameters. For individual objects, as is relevant for our study, the wind velocity may nonetheless be expected to "flip-flop", especially in the domain of LBVs (e.g. Pauldrach \& Puls 1990; Vink \& de Koter 2002).

\section{Results}

In the first part of this section, we compare our model to the results of a similar study by Li et al. (2000). Next, the effects of the various input parameters are discussed one-by-one. By constraining various stellar parameters to those of the well-studied, polarimetrically-variable $\mathrm{P} \mathrm{Cyg}$, the more detailed parameterspace is explored.

\subsection{Comparison with previous work}

$\mathrm{Li}$ et al. (2000) investigated the polarimetric variability of WolfRayet stars (WRs). Figure 5 shows a comparison between their results and the results of our model. Qualitatively the two studies show a similar trend of steadily-falling $\langle P\rangle$ with increasing $\mathcal{N}$, behaviour also found by Richardson et al. (1996). However, quantitatively the two differ by a factor of $\sim 5$. The reason for this is their different treatment of the clump density.

$\mathrm{Li}$ et al. fixed the clump angular size $\Delta \Omega$, clump thickness $\Delta r$, and the electrons per clump $N_{\mathrm{e}}$ at constant values. Therefore, the volume of the clump $V_{\mathrm{cl}}$ should increase with the square of the distance,

$V_{\mathrm{cl}} \propto r^{2}$.

They also stated that, following mass continuity, they scaled their electron density as

$n_{\mathrm{e}} \propto 1 /\left(r^{2} v\right)$.

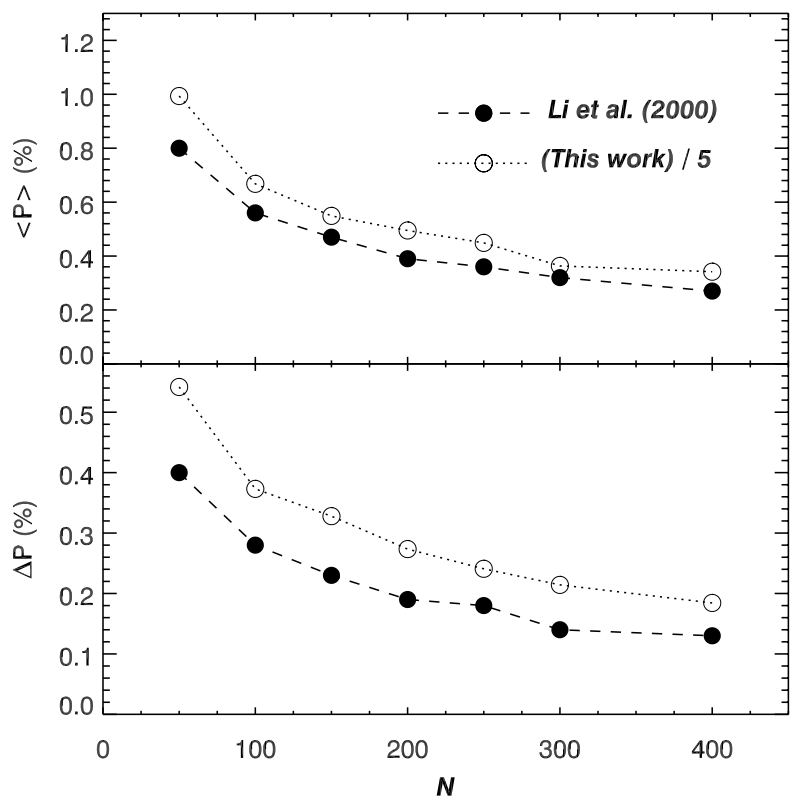

Fig. 5. Comparison between the results of $\mathrm{Li}$ et al. (2000) and the results of this work when divided by a scaling factor of 5. The top panel shows the time-averaged polarization as a function of clump ejections per wind flow-time, whilst the bottom panel shows the variability in $P$. The results of the present model have been scaled to highlight the fact that while the two models agree qualitatively, quantitatively they differ. Parameters used are $v_{\infty}=1800 \mathrm{~km} \mathrm{~s}^{-1}, R_{\star}=10 R_{\odot}, b=0.995$, and $\log \left(\dot{M} / M_{\odot} \mathrm{yr}^{-1}\right)=-4$.

These statements are not consistent: if the number of electrons per clump $N_{\mathrm{e}}=n_{\mathrm{e}} V_{\mathrm{cl}}$, then from Eq. (20),

$N_{\mathrm{e}} \propto 1 /\left(n_{\mathrm{e}} v\right)$.

Clearly, the number of electrons cannot be both constant and proportional to $1 / v$. For the system of equations to be selfconsistent, the clumps must either,

i) be allowed to grow in thickness as they propagate through the wind, due to the differential acceleration between the front and back of the clumps; or

ii) have a fixed thickness and an internal clump density which scales as $1 / r^{2}$. The space density of the clumps still falls as $1 / r^{2} v$.

Li et al. integrated from $\left(x_{1}=x^{\prime}\right) \rightarrow\left(x_{2}=x^{\prime}+\Delta r\right)$, where $\Delta r$ was constant, whilst allowing the electron density to fall as $1 / r^{2} v$. They therefore only integrated over the front $\Delta r$ of the clump, systematically underestimating the polarization per clump. This explains their lower levels of $\langle P\rangle$ compared to ours.

The differences in polarization per clump as a function of distance from the star is illustrated in Fig. 6. Three scenarios are plotted:

1 Clump density $\propto 1 / r^{2}$, with fixed $\Delta \Omega, \Delta r$.

2 Clump density $\propto 1 / r^{2} v$, with fixed $\Delta \Omega, \Delta r$.

3 Clump density $\propto 1 / r^{2} v$, with fixed $\Delta \Omega$, but with $\Delta r$ growing proportional to $v$.

It can be seen that there is a significant difference between scenario (2) and the self-consistent scenarios (1) and (3). Quantitatively, the difference between the results of $\mathrm{Li}$ et al. and this work can be understood in terms of the maximum polarization per clump $P_{\max }$. In the limit where the ejection rate is 


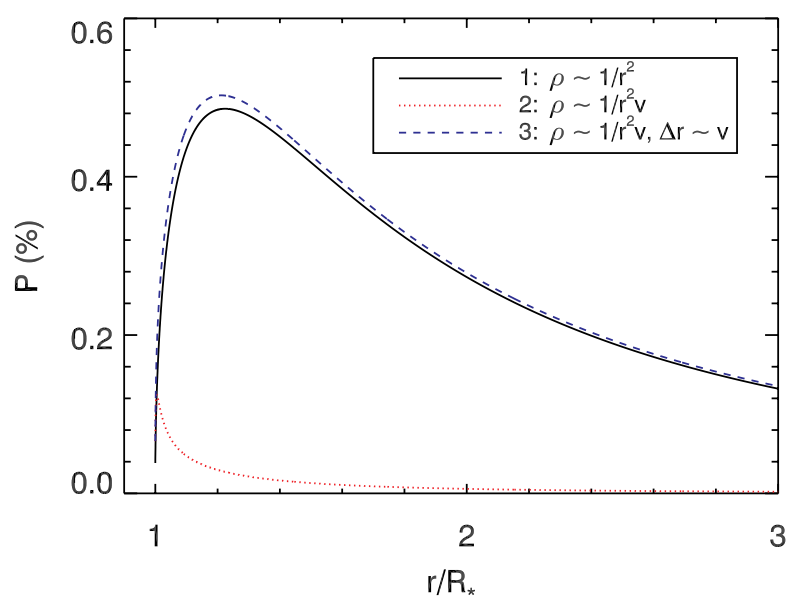

Fig. 6. Comparison between the three scenarios described in the text for calculating polarization as a function of distance for a single clump. Scenarios (1) and (3) are self-consistent and produce almost identical results, while (2) is different. Curve (2) peaks at $\sim 0.1 \%$ extremely close to the star, while (1) and (3) peak at $\sim 0.5 \%$ at a distance of $1.2 R_{\star}$. It is speculated that it is this factor of 5 difference in maximum polarization per clump which is responsible for the differing model results (see Fig. 5). The curves are evaluated for $\tau=1, \sin ^{2} \chi=1, \Delta \Omega=0.04$, and $b=0.995$.

much shorter than a flow-time, i.e. $\mathcal{N} \gg 1$, the time-averaged polarization $\langle P\rangle$ is proportional to $P_{\max }$. The maximum polarization per clump in scenario (2) is $\sim 0.1 \%$, while for (1) and (3) $P_{\max } \sim 0.5 \%$. This difference could explain the factor of $\sim 5$ difference between this work and $\mathrm{Li}$ et al. for this set of model parameters.

It can also be seen from Fig. 6 that polarimetrically there is little difference between scenarios (1) and (3). This is to be expected, as both scenarios integrate over the same amount of material and have the same optical depth. The minor difference when $x$ is small is due to the finite star correction term. The physical differences between scenarios (1) and (3) are as follows: (1) represents a thin shell of material being lifted off the surface of the star; while (3) describes a localised density overenhancement, the material of which moves through the wind in the same manner as the ambient material. We note that the two scenarios have very different implications for the clump fillingfactor $f_{\mathrm{cl}}$ : (3) has $f_{\mathrm{cl}}$ constant at all radii; while (1) has $f_{\mathrm{cl}}$ which grows with distance from the star to some asymptotic value. Figure 6 shows that while the two scenarios differ in the physical situation they describe, the resulting polarization is almost identical. As the computation times for (1) are much shorter, and integrals easier, this scenario is preferred throughout this study.

\subsection{Effect of various input parameters}

The free parameters in the model are the clump angular size $\Delta \Omega$, ejection rate $\mathcal{N}$, and the stellar parameters $\mu_{\mathrm{e}}, R_{\star}, \dot{M}, v_{\infty}$, and $v_{0}$ (i.e. $b$ ). Of these, $\Delta \Omega, \mu_{\mathrm{e}}$ and $\dot{M}$ will be fixed throughout the parameter study, so the effect of these variables is discussed below.

\subsubsection{Mass-loss rate}

The effect of varying $\dot{M}$ for a fixed set of model parameters is shown in Fig. 7. In the optically-thin limit, the polarization per clump is directly proportional to $\dot{M}$ (Eq. (9a)). Figure 7 shows, as expected, a linear relationship between $\dot{M}$ and $\langle P\rangle$ in the

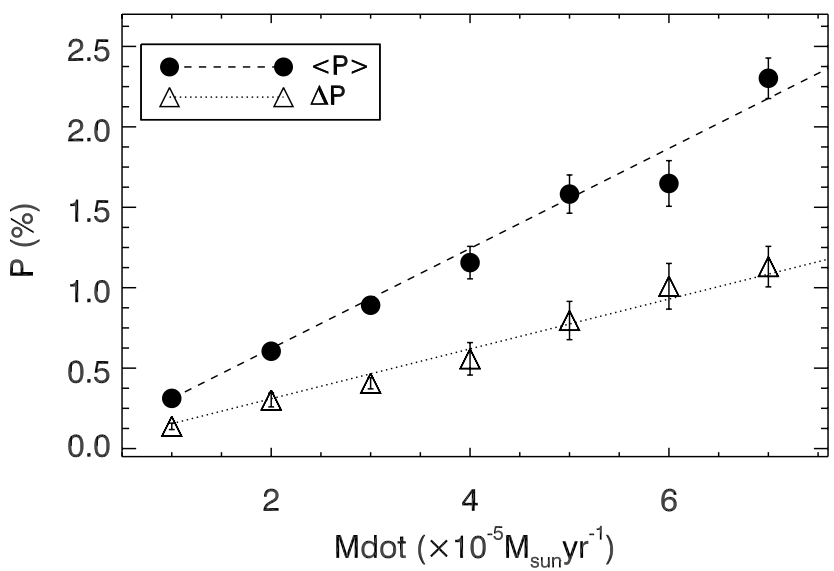

Fig. 7. The effect of varying mass-loss rate on the time-averaged polarization and its variability in the optically-thin regime. The model parameters used are $v_{\infty}=180 \mathrm{~km} \mathrm{~s}^{-1}, R_{\star}=100 R_{\odot}, \Delta \Omega=0.04, \Delta r=0.01$, $\mathcal{N}=100$.

optically-thin regime, and a linear least-squares-fit is overplotted to illustrate this. This linear relationship was also found by $\mathrm{Li}$ et al. (2000). As the mass-loss rate increases the clumps become optically-thick, therefore we would expect the trend to flatten-off for extremely-high mass-loss rates.

Also shown in Fig. 7 is the calculated polarimetric variability, $\Delta P$. This is also linear with $\dot{M}$, with $\Delta P /\langle P\rangle$ consistently found to be $\sim 0.5 \rightarrow 0.6$, not just as a function of mass-loss rate but for all simulations. This can be understood as follows: if $P$ is truly random, one would expect it to have the appearance of noise, with the amplitude $0 \rightarrow P_{\max }$ to be roughly $3 \sigma$. The timeaveraged value of $P$ will then be between these two at $\sim 1.5 \sigma$. Therefore, the quantity $\Delta P /\langle P\rangle \sim 1 / 1.5 \sim 0.67$.

\subsubsection{Clump size}

If the number of scatterers in a clump is conserved, then in theory changing the clump's size should have little effect, as the same amount of light will be scattered. However, there is a slight trend of decreasing polarization for larger clumps: consider a "shell" clump ejected perpendicular to the observer's line-ofsight. When viewed on the plane of the sky, light scattering towards the observer from the two opposing limbs of the clump will produce polarization with slightly different position-angles. For a clump with a large angular diameter, the position-angles of these two beams will tend toward being perpendicular to each other, thus we expect less net polarization from larger clumps. Quantitatively, for otherwise constant input parameters, from inspection of Eqs. (9a) and (9b) one would expect:

$\langle P\rangle \propto\left(\mu_{2}-\mu_{2}^{3}\right) / \Delta \Omega$

where $\mu_{2}=1-\Delta \Omega / 2 \pi$. The effect of varying $\Delta \Omega$ is shown in Fig. 8, with the relation in Eq. (22) scaled and overplotted. The behaviour of $\langle P\rangle$ follows this relation well, with the scatter due to the stochastic nature of the process.

Large values of $\Delta \Omega(\gtrsim 0.5)$ are likely to be physically unrealistic, and the fractional change in $\langle P\rangle$ between $\Delta \Omega=0.01 \rightarrow 0.5$ is small (Fig. 8). Therefore, we fix the angular size of the clumps to the value chosen by $\mathrm{Li}$ et al. (2000), that of $\Delta \Omega=0.04$. 


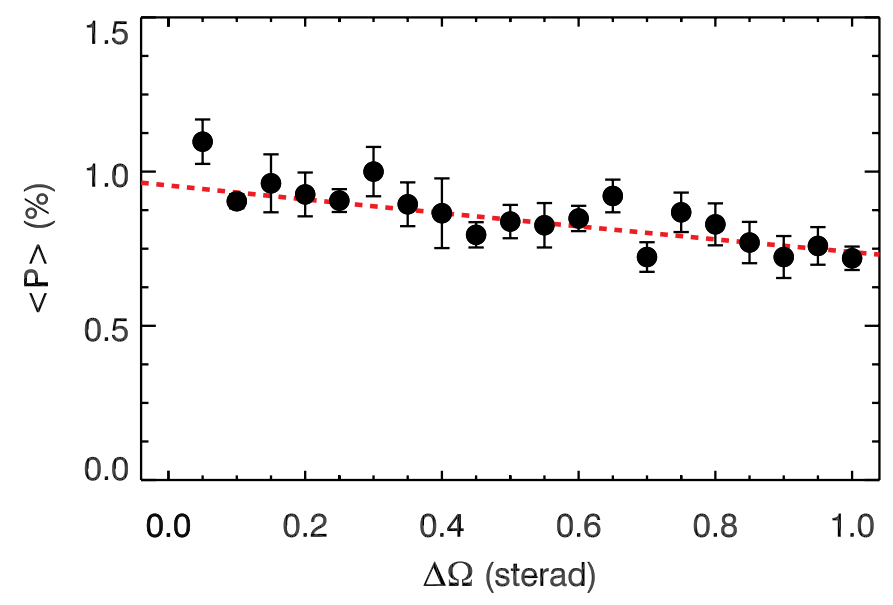

Fig. 8. The effect of varying clump angular size on the time-averaged polarization. Overplotted is the relation in Eq. (22), to which $\langle P\rangle$ is expected to be proportional (dashed line). Errors on the data points are from the uncertainties in the means of seven independent simulations. Input parameters are the same as used in Figs. 5 and 7.

\subsubsection{Mean mass-per electron}

The process considered in this study is electron-scattering, however the electrons in each clump contribute a negligible amount to the clump's mass. It is assumed that there are nuclei to account for each of these free electrons, e.g. if the wind material was pure $\mathrm{H}$ then the mass of a clump would be $N_{\mathrm{e}} m_{\mathrm{H}}$. However, the material in the winds of massive evolved stars will have a nonnegligible content of heavier elements, so the mass per clump is determined by the mean mass-per-electron, $\mu_{\mathrm{e}}$. From Eq. (9b) it can be seen that the optical depth (and hence the polarization per clump) is inversely proportional to $\mu_{\mathrm{e}}$. The reason for this is that greater values of $\mu_{\mathrm{e}}$ mean fewer electrons in a clump, reducing the electron-scattering optical depth.

The elemental abundances in a star's wind are determined to first order by the chemistry of the natal ISM and the evolutionary phase of the star. From recent abundance studies of hot supergiants it is assumed that the elements heavier than helium contribute a negligible amount to a clump's mass (e.g. Crowther et al. 2006). Determinations of the helium number fraction $Y=n_{\mathrm{He}} / n_{\mathrm{H}}$ are not straight-forward, due to subtle dependences of He line-strengths on the details of radiative transfer calculations (see e.g. McErlean et al. 1998). Studies of LBVs have yielded values of $Y=0.3-0.5$, (Crowther 1997; Najarro et al. 1997); while more recent studies of OB supergiants with upgraded models suggest slightly lower values $(Y=0.1-0.3$, Herrero et al. 2002; Repolust et al. 2005). This implies a value of $\mu_{\mathrm{e}} \sim 1.3-1.6$. As the uncertainty in the mean mass-per-electron is small compared to that of other parameters, a constant value of $\mu_{\mathrm{e}}=1.5$ is adopted throughout the study.

\subsection{Polarization as a function of stellar radius}

Following the above arguments, this section investigates the polarization produced by a clumpy wind across the range of stellar radii inferred from LBV temperatures. For a given mass and constant bolometric luminosity, the stellar radius and therefore effective temperature and terminal velocity can be varied. Then, for a given mass-loss rate, the clump ejection timescale and optical depth can be varied, and the resulting polarization calculated. Values of $L=10^{6} L_{\odot}, M=30 M_{\odot}$, and $\log \left(\dot{M} / M_{\odot} \mathrm{yr}^{-1}\right)=$ -4.5 are used, which are typical of the LBV AG Car
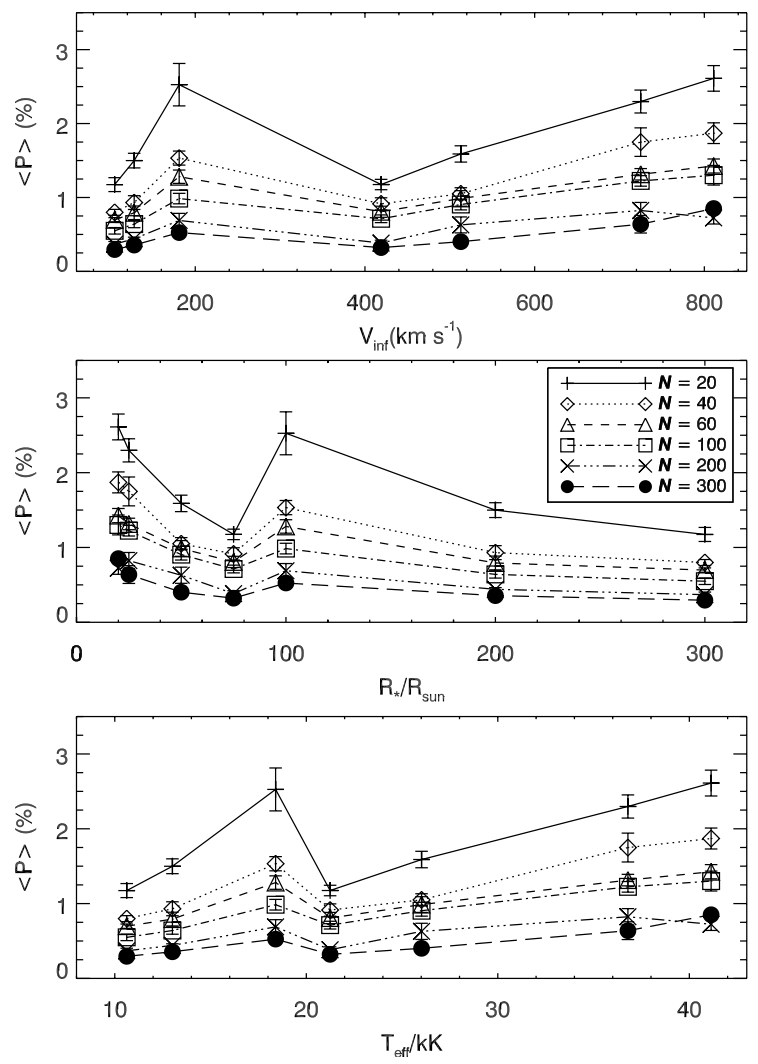

Fig. 9. Time averaged polarization for constant values of $\mathcal{N}$, as a function of terminal wind speed (top), $R_{\star}$ (middle), and effective temperature (bottom). The stellar parameters are the same as those used throughout the parameter study, namely $L=10^{6} L_{\odot}, M=30 M_{\odot}$, and $\log \left(\dot{M} / M_{\odot} \mathrm{yr}^{-1}\right)=-4.5$.

(Vink \& de Koter 2002). The effect of varying $\dot{M}$ is significant, as it is proportional to the time-averaged polarization (see previous section). $L$ and $M$, in this parameterization, only affect the terminal wind velocity, and so are redundant parameters if $v_{\infty}$ is known. The polarization as a function of $v_{\infty}, R_{\star}$ and $T_{\text {eff }}$ for a range of ejection timescales $\mathcal{N}$ is shown in Fig. 9.

\subsubsection{Varying ejection timescale}

If the mass-loss rate is conserved, increasing the number of clumps ejected per wind flow-time $\mathcal{N}$ will decrease the optical depth per clump. For low $\mathcal{N}$, the wind will consist of a small number of dense clumps. For increasing $\mathcal{N}$, the wind consists of a larger number of clumps with smaller optical depths, until eventually the wind tends toward a smooth, spherically symmetric outflow. Figure 9 illustrates this. It can be seen that while the level of polarization decreases with increasing $\mathcal{N}$, even at a high ejection rate there is still a detectable level of polarization $(>0.1 \%)$ for the wind parameters used.

\subsubsection{Varying clump optical depth}

For constant clump size, decreasing the initial optical depth per clump will also decrease the mass per clump. Therefore if the mass-loss rate is to be conserved the ejection rate must increase. The wind will be highly clumped at $\tau_{0}=1$, and will tend towards 
a smooth outflow at low values of $\tau$. For the model parameters of Fig. 9, $\mathcal{N}$ is linearly related to $\tau$ via,

$\tau=3.6 \times 10^{5} \mathcal{N}^{-1}\left(\frac{v_{\infty}}{\mathrm{km} \mathrm{s}^{-1}}\right)^{-1}\left(\frac{R_{\star}}{R_{\odot}}\right)^{-1}$.

Evaluating this expression for the lowest curves in Fig. 9, optical depths are in the region $\tau_{0}=0.05 \rightarrow 0.1$. As $v_{\infty} \propto 1 / \sqrt{R_{\star}}$ (Eq. (17)), optical depths are highest for small radius, high terminal wind-speed stars.

\subsubsection{Overall behaviour of $P$ with $R_{\star}$}

It can be seen from Fig. 9 that, for a given ejection-rate per wind flow-time there is a general trend of increasing polarization with decreasing radius. This can be understood as follows: as the size of the star is decreased, the clumps become smaller and denser, producing more polarization per clump. Around the bi-stability zone, the terminal velocity increases, and clumps spend less time close to the star: the overall polarization drops again.

The overall levels of polarization produced in the simulations are high - even at high $\mathcal{N} \sim 300$ where the wind is tending toward a smooth outflow, observable residual levels of polarization $(\sim 0.3 \%)$ are produced. This is discussed further in Sect. 5 .

\subsection{Optically-thick clumps}

In this and the following section, the parameter-space is explored in greater depth. To do this, the mass-loss rate, stellar radius and terminal wind-speed are fixed at those of the LBV P Cyg, the star for which most polarimetric observations exist. Hayes (1985), Taylor et al. (1991) and Nordsieck et al. (2001) each present many individual measurements, finding $\langle P\rangle \simeq 0.3 \pm 0.15 \%$. Indeed, it is an extremely well studied object generally, with many independent measurements of stellar/wind parameters in the literature. As the star hasn't been significantly variable for $\gtrsim 200$ years, this means that the input parameters of stellar radius, mass-loss rate and terminal wind speed can be constrained. This then allows further exploration of other details which may or may not be significant.

The parameters used are those derived by Najarro et al. (1997), namely

$R_{\star} / R_{\odot}=75, v_{\infty}=185 \mathrm{~km} \mathrm{~s}^{-1}, \log \left(\dot{M} / M_{\odot} \mathrm{yr}^{-1}\right)=-4.52$.

These values were found through spectroscopic analysis with non-LTE modelling. The model took no account of windclumping, therefore the mass-loss rate may be an overestimate. Also, the authors note that there is some degeneracy between $R_{\star}, v_{\infty}$ and $\dot{M}$ such that $\dot{M} /\left(v_{\infty} R_{\star}^{1.5}\right) \sim$ constant, but constrain $R_{\star}$ through other derived values in the literature.

Figure 10 shows the time-averaged polarization over a broader range of $\mathcal{N}$ than in Fig. 9. At high $\mathcal{N}$, the static model was used to calculate $P$, and it can be seen that the level of polarization observed for P Cyg is reached at $\mathcal{N} \sim 2000$.

At low $\mathcal{N}$, the density-per-clump becomes very high in order to conserve the mass-loss rate, and exceeds unity below $\mathcal{N} \sim 20$. At this point, the model switches to the optically-thick approximation, that is the polarization per clump plateaus at $\tau>1$. Therefore at $\mathcal{N}<20$ the overall polarization begins to fall again. We again point out that, in the optically-thick regime, the polarization may become sensitive to clump morphology, and hence the optically-thick part of the curve in Fig. 10 (left of $\mathcal{N} \simeq 10$ ) may not be as quantitatively robust as the thin-clump regime.

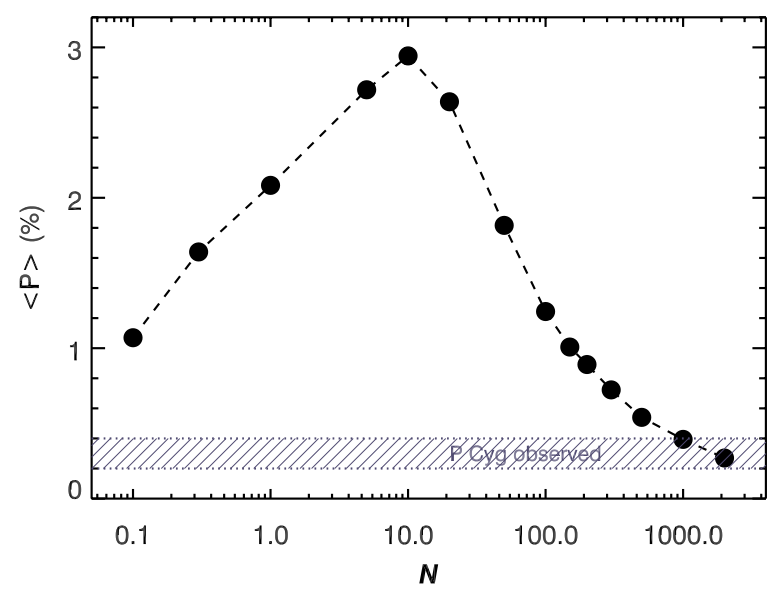

Fig. 10. Time-averaged polarization over a broad range of $\mathcal{N}$. At $\mathcal{N} \sim$ 20 , the optical depth per clump exceeds unity, and the overall polarization falls (see text for details). The P Cyg wind parameters derived by Najarro et al. (1997) are used. The observed level of polarization for P Cyg is marked by the dash-dotted line.

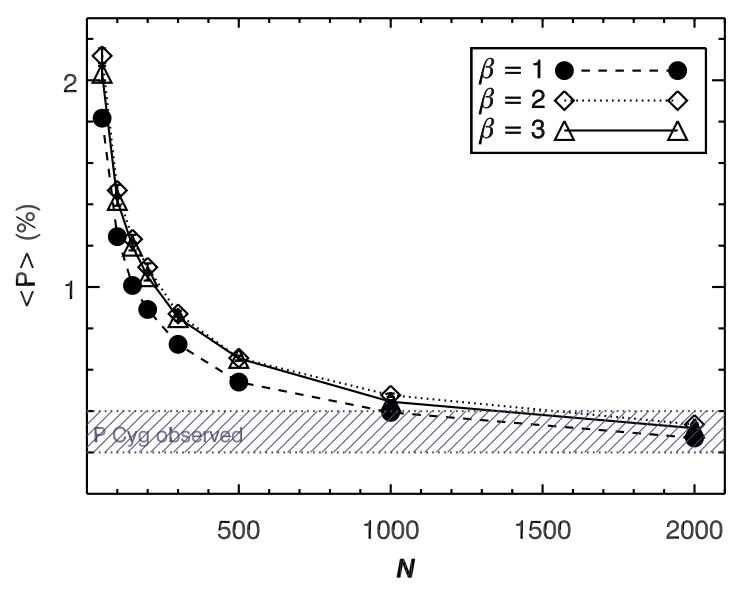

Fig. 11. The polarization produced when the wind acceleration parameter $\beta$ is increased, lowering the acceleration of the wind. Stellar parameters are the same as Fig. 10.

However, the qualitative behaviour of a turnover in $\langle P\rangle$ is inevitable, as there is only a maximum amount of polarization a single clump can produce. Therefore, we expect that as clump density increases and the number of clumps becomes smaller, there will be two solutions to match the observed level of polarization - the optically-thin, and optically-thick cases.

Extrapolating from Fig. 10, the optically-thick solution for $\mathrm{P}$ Cyg is reached at at $\mathcal{N} \lesssim 0.01$. As the flow-time for P Cyg is about 3 days, this would imply ejection rates of only a few per year even allowing for a factor of 10 difference in the calculations. This is deemed to be unlikely, for reasons which will be discussed in greater detail in Sect. 5, and we therefore favour the optically-thin solution.

\subsection{Lower wind acceleration}

In Fig. 11 the effect of increasing the wind acceleration parameter $\beta$ is explored. It can be seen that, in the case of $\mathrm{P} \mathrm{Cyg}$, increasing $\beta$ makes very little difference. This is related to the ratio $v_{0} / v_{\infty}$ : from Fig. 3 , it is clear that the greatest amount of polarization is produced when the clump is within $\sim 6 R_{\star}$ of the 
star. Figure 4 shows that when $v_{0} / v_{\infty}$ is relatively large, altering $\beta$ makes virtually no difference to the dwell-time. Only when $v_{0} / v_{\infty}$ is small is the dwell-time significantly affected by $\beta$. It is noted that at larger $v_{\infty}$, increasing $\beta$ has a more pronounced effect, as would be expect from the low $v_{0} / v_{\infty}$ case shown in Fig. 4. At $v_{0} / v_{\infty} \sim 1 / 180$, the polarization is increased by a factor of $\sim 1.5$ when $\beta$ is increased to 2 .

\section{Discussion}

\subsection{A few massive clumps or many tiny clumps?}

In the approximation of a wind consisting of uniform clumps with no inter-clump medium, the densities of the individual clumps need not be large to produce the levels of polarization observed in massive stars. Polarimetric variability on the scale observed in LBVs can in principle be reproduced by two distinct regimes of $\mathcal{N}$ : either $\lesssim 0.1$ in the optically-thick case, or $\gtrsim 1000$ in the optically-thin case (see Fig. 10).

In the former, the time between ejections would be of the order of months (the flow-time of P Cyg is about 3 days), and one would expect ejection events to be seen in the data of Hayes (1985) whose temporal resolution was of order 1 day. Also, the density of the clumps would be so large that the recombination emission would almost certainly be conspicuous in timeresolved spectroscopy (quantitative analysis of this is beyond the scope of the present work). For this reason, the higher value of $\mathcal{N}$ is considered to be the most likely value of the two.

At the inferred ejection rates of $\mathcal{N} \gtrsim 10^{3}$, the inner wind consists of thousands of low density clumps. In this regime, one may expect the clumps to cancel each other out and leave a net polarization of zero. However, only a slight imbalance is required to produce residual polarization. For example, at $\mathcal{N}=2000$ and the stellar parameters of $\mathrm{P} \mathrm{Cyg}$, the maximum polarization per clump is $0.006 \%$, and there are $\sim 7 \times 10^{3}$ clumps in the inner $2 R_{\star}$. A Poissonian $1 \sigma$ deviation from spherical symmetry of $\sim 80$ clumps is enough to produce a residual polarization of $\langle P\rangle=0.4 \%$, which is the observed level of P Cyg. Therefore, the polarization may simply result from the statistical deviations from spherical symmetry: a slightly-fragmented wind model.

If observable levels of polarization are produced when a wind is so close to being homogeneous, it is reasonable to ask why intrinsic polarization is not present in all the stars observed by (Davies et al. 2005). The answer may be related to the star's mass-loss rate: as shown in Fig. 7, the polarization produced by a given simulation is proportional to $\dot{M}$ if all other parameters are fixed. Therefore, if a star's ejection rate were fixed but its massloss rate were reduced from $10^{-4.5}$ to $10^{-6} M_{\odot} \mathrm{yr}^{-1}$, its average polarization could go from being as large as any observed to being completely undetectable. This suggests that intrinsic polarization may be ubiquitous among hot stars with mass-loss rates greater than $10^{-4.5} M_{\odot} \mathrm{yr}^{-1}$, as a measurable level of polarization can be produced from a statistical deviation from spherical symmetry.

This conclusion is supported by the findings of Davies et al. (2005). Intrinsic polarization is more likely to be found in stars with strong $\mathrm{H} \alpha$ emission; and for LBVs, whose terminal wind velocities are roughly the same, $\mathrm{H} \alpha$ equivalent width may be considered to be a crude measure of mass-loss rate. It is also consistent with the lower incidence of polarization in O-supergiants (Osgs) and WRs, found in Harries et al. (1998, 2002). Osgs have typical mass-loss rates of $\lesssim 10^{-5} M_{\odot} \mathrm{yr}^{-1}$ (Puls et al. 2006); and while WRs are considered to have $\dot{M} \sim 10^{-5} \rightarrow 10^{-4} M_{\odot} \mathrm{yr}^{-1}$, ironically this may be reduced due to the effect of clumping
(Hamann \& Koesterke 1998). The impact of clumping on LBV mass-loss rates is yet to be studied with the latest versions of model atmospheres.

Of course, there is the possibility that the wind is a combination of the two scenarios, where a slightly fragmented wind produces low-level variability, combined with sporadic ejections of optically-thick clumps. Such a wind could be simulated by replacing the uniform clumps of the present model with clumps whose optical depths are determined by a power-law distribution. This would involve the introduction of an extra free parameter, the power-law index, and so it would be reasonable to look for observational evidence for such a scenario first before increasing the degenerate parameter space. The polarimetric signature of a dominant dense clump would be a jump in polarization and a PA which remained constant for several flow-times (few weeks for P Cyg), until its effect is "washed-out" by the smaller clumps close to the star. Also, it was shown by Ignace \& Churchwell (2004) that hierarchical clumping of ionized outflows can lead to atypical spectral indices in IR and radio freefree emission. Such spectra have slopes intermediate between the optically-thick/thin limits over several orders of magnitude in frequency. Hence, a combination of polarimetric monitoring and dynamic modelling, plus continuum radio observations, may be used to investigate hierarchical wind-clumping further.

\subsection{Wind "clumping factor"}

The clumping of hot star winds has been the subject of much recent interest, as it has become apparent that a clumped wind can lead to increased emission for the same amount of material. This means that mass-loss rates derived from spectroscopic analysis under the assumption of a smooth outflow are systematically over-estimated by factors of 3 and higher (Bouret et al. 2005; Fullerton et al. 2006; Puls et al. 2006). To quantify this, the clumping of a star's wind is usually parameterized by defining the wind clumping factor as a function of the time-averaged wind density,

$f_{\mathrm{cl}}=\frac{\langle\rho\rangle^{2}}{\left\langle\rho^{2}\right\rangle}$

in the limit where there is negligible inter-clump medium. The clumping-factor itself is usually assumed to be a function of wind-speed (and hence distance from star),

$f_{\mathrm{cl}}=f_{\infty}+\left(1-f_{\infty}\right) \exp \left(-v / v_{\mathrm{cl}}\right)$

such that the wind starts off smooth, and clumping is "switched on" at some scale-height above the photosphere when the velocity reaches $v_{\mathrm{cl}}$. When incorporating clumping into ionization models, some studies have chosen $v_{\mathrm{cl}}$ such that clumping becomes important at large distances from the star (e.g. Dessart et al. 2000), where hydrodynamical models have shown that clumping naturally arises from instabilities in the line-driving mechanism (Runacres \& Owocki 2002). More contemporary studies have chosen smaller values of $v_{\mathrm{cl}}$ to initiate clumping just above the photosphere, based on the effect a given clumpinglaw has on spectral line profiles (Hillier et al. 2003). More recently still, Puls et al. (2006) presented an investigation into the clumping factor of different regions of the wind using simultaneous optical, IR and radio observations. They find that, for stars with winds dense enough to produce line-emission, the wind is strongly clumped in the inner $2 R_{\star}$, where the line-emission is produced. 
A direct determination of the clumping-law is not possible from polarimetry alone. The polarization produced by a clump depends on the amount of light the clump intercepts, and the thickness of the clump is a redundant parameter provided the column-density remains constant. Hence, polarimetry cannot distinguish between a dense, flattened clump and a less-dense, extended clump, as both have the same optical depth.

However, the aspects of wind-structure probed by polarimetry are useful in conjuction with other observations in determining the filling-factor of the wind. It has been shown here that polarimetry is sensitive to the number of clumps that the wind is broken up into. This may be relevant to other recent studies of wind-structure, in particular the study of the X-ray line-profiles.

Luminous, O-type supergiants have been observed to emit X-ray line-emission (e.g. Cassinelli \& Swank 1983), likely due to shocked gas embedded in their outflowing winds (Lucy \& White 1980; Owocki et al. 1988). In models of such features the red-shifted emission from the back-hemisphere, which has a larger path-length to travel to the observer, is predicted to be attenuated by the intervening material, producing asymmetric line profiles (Owocki \& Cohen 2001; Ignace \& Gayley 2002). However, this is not observed, with the X-ray line profiles of Osgs remarkably symmetric in appearance (e.g. Schulz et al. 2000; Waldron \& Cassinelli 2001).

In order to explain this phenomenon, it has been suggested that either the mass-loss rate must be reduced by a factor of $\gtrsim 5$ (Cohen et al. 2006), or that the wind must be clumped in such a way as to make the overall wind very porous, i.e. there must be large separation between the clumps for the photons to leak through (Feldmeier et al. 2003; Owocki \& Cohen 2006). The porosity-length, $h$, is defined as the ratio of the clump size to the volume filling-factor, so having a large value of $h$ sets the inter-clump distance to be very large compared to the size of each clump. In this regime, the X-rays emitted from the back hemisphere can travel through the wind relatively unattenuated, and a symmetric line-profile is recovered.

Polarimetry can be used to constrain the available parameterspace of these solutions. In the porous-wind model of Owocki \& Cohen (2006), there is degeneracy between clump size and filling-factor - that is, the wind can consist of a few, large clumps with a large volume filling-factor; or a large number of small, dense clumps and very small filling-factor. These two regimes would produce very different polarimetric signatures. In a wind containing very few clumps, the polarimetric evolution of individual clumps could be traced, providing there were only a handful of clumps in the inner $\sim 5 R_{\star}$. Resolving the dynamical evolution of individual clumps would imply that the number of clumps was small, and we could then place a lower-limit on the filling-factor for a given porosity-length.

If significant intrinsic polarization was detected, but no individual clumps were resolved, this would allow us to place a lower-limit to the number of clumps, and hence an upper-limit to $f$. Were no intrinsic polarization to be observed at all, this would imply from our models that the number of clumps so large that the wind was tending towards a smooth outflow, which would not be consistent with asymmetric X-ray line profiles. Given that $\langle P\rangle$ scales with $\dot{M}$ in our models, such a situation may instead be consistent with a lower mass-loss rate for that object.

\section{Summary}

We present an analytic investigation into the wind clumping of hot stars, from the point-of-view of their polarimetric variability.
In particular, we study the effect of varying stellar parameters, with the aim of reproducing the level and variability observed in LBVs.

The parameter space was defined such that the only key input parameter for a given set of stellar/wind parameters was the ejection timescale. It was found that, to quantitatively reproduce the polarimetric variability of hot stars, the ejection timescale must either be very short ( $\ll 1$ per hour) or very long ( $\sim$ few per year). In the latter case, the polarization arises from the sporadic ejection of very dense clumps, and the variability timescale is very long. In the former, the inner wind consists of thousands of low-density clumps, with the observed polarization resulting from random statistical deviations from spherical symmetry in a slightly-fragmented wind. Here, significant changes are expected in night-to-night monitoring. As the polarization of hot stars is observed to be variable on very short timescales, and as they are not associated with any significant spectroscopic variability that would arise from dense clumps, the slightly-fragmented wind explanation is favoured.

As polarization scales linearly with mass-loss rate, it is speculated that all hot stars with high mass-loss rates are likely to display polarimetric variations. This is consistent with the results of Davies et al. (2005) which reveal that intrinsic polarization is more likely to be found in those LBVs with the strongest $\mathrm{H} \alpha$ emission, which is a rough indicator of mass-loss rate. It is also consistent with the lower incidence of polarization in $\mathrm{O}$ supergiants and WRs, which likely have mass-loss rates factors of $\gtrsim 5$ lower.

Acknowledgements. We would like to thank the referee Rico Ignace and editor Steven Shore for their insightful suggestions and comments at the refereeing stage, which improved the paper. We also thank John Brown, Qingkang Li, Stan Owocki and Joachim Puls for fruitful discussions during the course of this work. BD aknowledges funding by PPARC. J.S.V. acknowledges RCUK for his academic fellowship.

\section{References}

Bouret, J.-C., Lanz, T., \& Hillier, D. J. 2005, A\&A, 438, 301 Brown, J. C., \& McLean, I. S. 1977, A\&A, 57, 141

Brown, J. C., Ignace, R., \& Cassinelli, J. P. 2000, A\&A, 356, 619

Cassinelli, J. P., \& Swank, J. H. 1983, ApJ, 271, 681

Cassinelli, J. P., Nordsieck, K. H., \& Murison, M. A. 1987, ApJ, 317, 290

Code, A. D., \& Whitney, B. A. 1995, ApJ, 441, 400

Cohen, D. H., Leutenegger, M. A., Grizzard, K. T., et al. 2006, MNRAS, 368, 1905

Crowther, P. A. 1997, in Luminous Blue Variables: Massive Stars in Transition, ed. A. Nota, \& H. Lamers, ASP Conf. Ser., 120, 51

Crowther, P. A., Lennon, D. J., \& Walborn, N. R. 2006, A\&A, 446, 279

Davies, B., Oudmaijer, R. D., \& Vink, J. S. 2005, A\&A, 439, 1107

Dessart, L., Crowther, P. A., Hillier, D. J., et al. 2000, MNRAS, 315, 407

Feldmeier, A., Oskinova, L., \& Hamann, W.-R. 2003, A\&A, 403, 217

Fullerton, A. W., Massa, D. L., \& Prinja, R. K. 2006, ApJ, 637, 1025

Hamann, W.-R., \& Koesterke, L. 1998, A\&A, 335, 1003

Harries, T. J. 2000, MNRAS, 315, 722

Harries, T. J., Hillier, D. J., \& Howarth, I. D. 1998, MNRAS, 296, 1072

Harries, T. J., Howarth, I. D., \& Evans, C. J. 2002, MNRAS, 337, 341

Hayes, D. P. 1984a, AJ, 89, 1219

Hayes, D. P. 1984b, ApJS, 55, 179

Hayes, D. P. 1985, ApJ, 289, 726

Herrero, A., Puls, J., \& Najarro, F. 2002, A\&A, 396, 949

Hillier, D. J., Lanz, T., Heap, S. R., et al. 2003, ApJ, 588, 1039

Ignace, R., \& Churchwell, E. 2004, ApJ, 610, 351

Ignace, R., \& Gayley, K. G. 2002, ApJ, 568, 954

Kudritzki, R.-P., \& Puls, J. 2000, ARA\&A, 38, 613

Lamers, H. J. G. L. M., Snow, T. P., \& Lindholm, D. M. 1995, ApJ, 455, 269

Li, Q., Brown, J. C., Ignace, R., Cassinelli, J. P., \& Oskinova, L. M. 2000, A\&A, 357,233

Lucy, L. B., \& White, R. L. 1980, ApJ, 241, 300

Lupie, O. L., \& Nordsieck, K. H. 1987, AJ, 93, 214 
Machado, M. A. D., de Araújo, F. X., Pereira, C. B., \& Fernandes, M. B. 2002 , A\&A, 387, 151

McErlean, N. D., Lennon, D. J., \& Dufton, P. L. 1998, A\&A, 329, 613

Melgarejo, R., Magalhães, A. M., Carciofi, A. C., \& Rodrigues, C. V. 2001, A\&A, 377, 581

Mokiem, M., de Koter, A., Vink, J., \& Puls, J. E. A. 2007, A\&A

Najarro, F., Hillier, D. J., \& Stahl, O. 1997, A\&A, 326, 1117

Nordsieck, K. H., Wisniewski, J., Babler, B. L., et al. 2001, in P Cygni 2000: 400 Years of Progress, ed. M. de Groot, \& C. Sterken, ASP Conf. Ser., 233

Oskinova, L., Feldmeier, A., \& Hamann, W.-R. 2006, A\&A, 372, 313

Owocki, S., \& Cohen, D. 2001, ApJ, 559, 1108

Owocki, S., \& Cohen, D. 2006, ApJ, 648, 565

Owocki, S. P., Castor, J. I., \& Rybicki, G. B. 1988, ApJ, 335, 914

Pauldrach, A. W. A., \& Puls, J. 1990, A\&A, 237, 409

Puls, J., Markova, N., Scuderi, S., et al. 2006 [arXiv: astro-ph/0604372]

Repolust, T., Puls, J., \& Herrero, A. 2004, A\&A, 415, 349

Repolust, T., Puls, J., Hanson, M. M., Kudritzki, R.-P., \& Mokiem, M. R. 2005, A\&A, 440, 261

Richardson, L. L., Brown, J. C., \& Simmons, J. F. L. 1996, A\&A, 306, 519

Robert, C., Moffat, A. F. J., Bastien, P., Drissen, L., \& St.-Louis, N. 1989, ApJ, 347,1034
Rodrigues, C. V., \& Magalhães, A. M. 2000, ApJ, 540, 412

Runacres, M. C., \& Owocki, S. P. 2002, A\&A, 381, 1015

Schulte-Ladbeck, R. E., Clayton, G. C., Hillier, D. J., Harries, T. J., \& Howarth, I. D. 1994, ApJ, 429, 846

Schulte-Ladbeck, R. E., Leitherer, C., Clayton, G. C., et al. 1993, ApJ, 407, 723 Schulz, N. S., Canizares, C. R., Huenemoerder, D., \& Lee, J. C. 2000, ApJ, 545, L135

St.-Louis, N., Drissen, L., Moffat, A. F. J., Bastien, P., \& Tapia, S. 1987, ApJ, 322,870

Stahl, O., Jankovics, I., Kovcs, J., et al. 2001, A\&A, 375, 54

Taylor, M., Nordsieck, K. H., Schulte-Ladbeck, R. E., \& Bjorkman, K. S. 1991, AJ, 102, 1197

van de Hulst, H. C. 1980, Multiple light scattering. Tables, formulas and applications (New York: Academic Press)

Vink, J. S., \& de Koter, A. 2002, A\&A, 393, 543

Vink, J. S., de Koter, A., \& Lamers, H. J. G. L. M. 1999, A\&A, 350, 181

Vink, J. S., de Koter, A., \& Lamers, H. J. G. L. M. 2000, A\&A, 362, 295

Waldron, W. L., \& Cassinelli, J. P. 2001, ApJ, 548, L45

Zickgraf, F.-J., \& Schulte-Ladbeck, R. E. 1989, A\&A, 214, 274 